\title{
Chronic Kidney Disease and Cognitive Impairment: A Systematic Review and Meta-Analysis
}

\author{
Thorleif Etgen ${ }^{\mathrm{a}, \mathrm{c}}$ Michel Chonchol ${ }^{\mathrm{e}}$ Hans Förstl ${ }^{\mathrm{a}}$ Dirk Sander ${ }^{\mathrm{b}, \mathrm{d}}$ \\ Departments of a Psychiatry and Psychotherapy, and ${ }^{b}$ Neurology, Technische Universität München, München, \\ 'Department of Neurology, Kliniken Südostbayern - Klinikum Traunstein, Traunstein, and d Department of \\ Neurology, Benedictus Krankenhaus Tutzing, Tutzing, Germany; ${ }^{e}$ Division of Renal Diseases and Hypertension, \\ University of Colorado School of Medicine, Aurora, Colo., USA
}

\section{Key Words}

Chronic kidney disease $\cdot$ Cognitive impairment •

Dementia $\cdot$ Risk factors

\begin{abstract}
Background: Chronic kidney disease (CKD) has emerged as a possible new risk factor of cognitive impairment and dementia, but results of studies remain conflicting. Methods: A systematic literature research of electronic databases (MEDLINE, Cochrane Library and Goggle Scholar covering the period from 1980 to January 2012) and meta-analysis of relevant cross-sectional and longitudinal studies were conducted to assess the association of CKD and cognitive decline. Results: Most cross-sectional and longitudinal studies suggest an association between cognitive impairment and CKD. Meta-analysis of cross-sectional and longitudinal studies comprising 54,779 participants yielded an association of cognitive decline in patients with CKD compared with patients without CKD (OR 1.65, 95\% Cl 1.32-2.05; $p<0.001$, and OR 1.39, 95\% Cl 1.15-1.68; $p<0.001$, respectively). Conclusion: This is the first meta-analysis assessing the impact of CKD on cognitive decline. Our results suggest CKD being a significant and independent somatic risk factor in the development of cognitive decline.
\end{abstract}

Copyright $\odot 2012$ S. Karger AG, Basel

\section{KARGER}

Fax +41613061234

E-Mail karger@karger.ch

www.karger.com
(C) 2012 S. Karger AG, Basel

0250-8095/12/0355-0474\$38.00/0

Accessible online at:

www.karger.com/ajn

\section{Introduction}

The worldwide incidence of persons affected by cognitive decline including cognitive impairment and dementia is increasing. Therefore, the early identification of possible precursors of dementia and the diagnosis and treatment of modifiable risk factors is of increased importance [1]. The concept of mild cognitive impairment which describes cognitive impairment beyond that of normal ageing but, in contrast to dementia, does not interfere notably with activities of daily life, permits timely identification of patients at high risk of developing dementia $[2,3]$. This concept opens a potentially larger therapeutic window and increases the significance of modifiable risk factors $[4,5]$.

In recent years, chronic kidney disease (CKD) has been proposed as an independent risk factor for cognitive decline but the available data are conflicting $[6,7]$. Whereas many cross-sectional and most of the longitudinal studies have demonstrated that CKD is associated with cognitive decline, some more recent prospective studies could not confirm this association. Therefore, the first aim of this study was to perform an up-to-date systematic literature review and search for possible differences among the various studies which might explain the

PD Dr. Thorleif Etgen

Department of Neurology, Kliniken Südostbayern - Klinikum Traunstein Cuno-Niggl-Strasse 3

DE-83278 Traunstein (Germany)

Tel. +49861 705 1771, E-Mail thorleif.etgen@klinikum-traunstein.de 
divergent results. The second aim was to conduct a metaanalysis of all available large cross-sectional and prospective studies assessing the association between CKD and cognitive decline.

\section{Material and Methods}

\section{Selection of Studies}

Studies that examined the association between CKD and cognitive decline were identified by a computerized research of electronic databases including MEDLINE, Cochrane Library and Goggle Scholar. This research included the period from 1980 to January 2012 and used the following Medical Subject Heading $(\mathrm{MeSH})$ terms 'chronic kidney disease', 'renal failure', 'cognitive impairment', 'cognitive decline' and 'dementia'. Manual review of the reference list of all retrieved articles was part of our research. We assessed all potentially relevant articles for eligibility. Inclusion or exclusion of studies was decided hierarchically on the basis of the (1) study title, (2) the abstract and (3) completeness of the study.

Studies were included if they fulfilled all following criteria: (1) either cross-sectional or longitudinal study, (2) at least 150 participants, (3) association of CKD and cognitive impairment as primary or secondary outcome and (4) results presented by odds ratio (OR) or hazard ratio (HR) values or sufficient data to calculate these parameters. The primary outcome for this meta-analysis was cognitive impairment (fig. 1).

\section{Statistical Analysis}

The meta-analysis was conducted separately for cross-sectional and longitudinal studies [8]. The meta-analysis to pool results from the individual studies was done with Review Manager (RevMan; version 5.1 for Macintosh; Copenhagen, Denmark). Pooled results are reported as OR and are presented with 95\% confidence interval (CI) with two-sided $\mathrm{p}$ values using a random-effects model. $\mathrm{p}<0.05$ was considered to be statistically significant. We used studies from multivariate models with the most complete adjustment for potential confounders. Statistical heterogeneity was evaluated using the $\mathrm{I}^{2}$ statistic, which assesses the appropriateness of pooling the individual study results. The $\mathrm{I}^{2}$ value provides an estimate of the amount of variance across studies because of heterogeneity rather than chance. Where $\mathrm{I}^{2}$ was greater than $50 \%$, heterogeneity was considered to be high. Moreover, to further investigate the heterogeneity across studies, we performed sensitivity analyses by dividing studies into groups according to their main characteristics. Subgroup analyses were then performed according to degree of CKD (estimated glomerular filtration rate $($ GFR) $>60$ vs. eGFR $45-60$ and eGFR $>60$ vs. eGFR $<45 \mathrm{ml} / \mathrm{min} / 1.73 \mathrm{~m}^{2}$ ), gender, mean sample size of the study populations (less than/at least 3,000), mean duration of follow-up (less than/at least 3 years) and method used to evaluate cognitive function (Mini-Mental State Examination (MMSE)/other). Publication bias was appraised by visual inspection of the funnel plot of effect size against standard error.

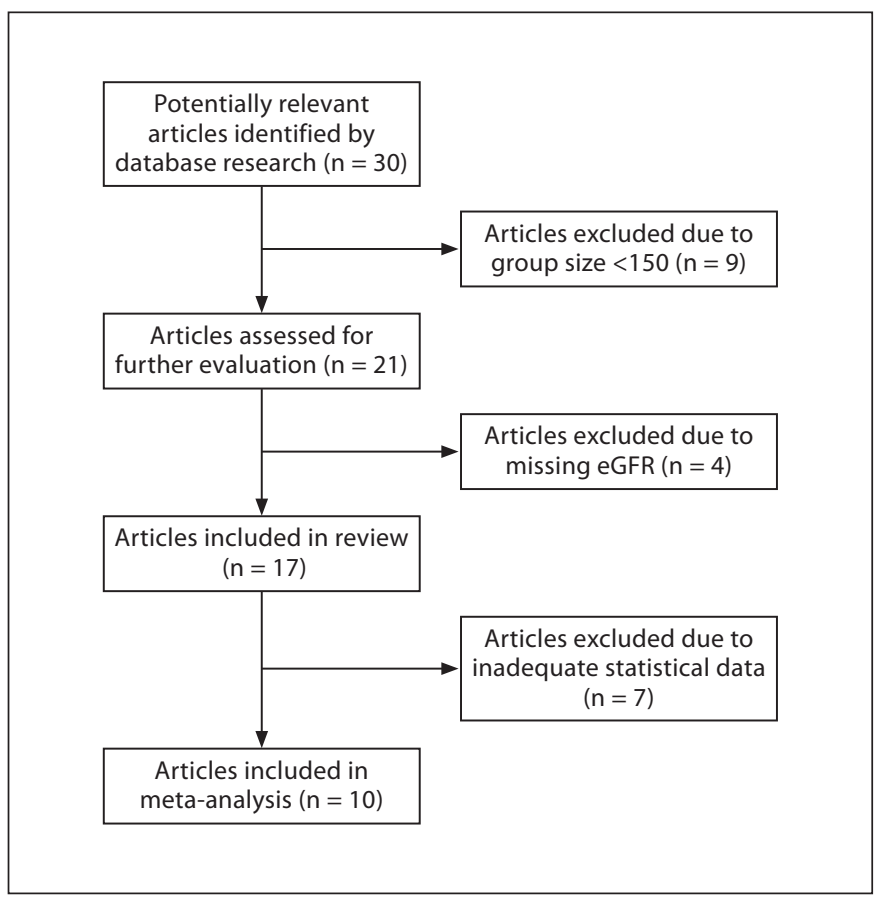

Fig. 1. Flow chart of search strategy.

\section{Results}

\section{Cross-Sectional Studies}

Seven entirely cross-sectional studies which examined the association between CKD and cognitive function were retrieved (table 1). In the Heart Estrogen/Progestin Replacement Study including 1,015 women with established coronary artery disease aged $<80$ years, an approximate $15-25 \%$ increase in risk for cognitive dysfunction per $10 \mathrm{ml} / \mathrm{min} / 1.73 \mathrm{~m}^{2}$ decrement in eGFR was observed [9]. In the Third National Health and Nutrition Examination Survey moderate CKD (eGFR 30-59 ml/ $\min / 1.73 \mathrm{~m}^{2}$ ) was significantly associated with poorer learning or concentration and impairment in visual attention [10]. The Reasons for Geographic and Racial Differences in Stroke Study (REGARDS) observed an increased prevalence of cognitive impairment among participants with CKD, independent of confounding factors [11]. In the Maine-Syracuse Longitudinal Study, participants with CKD presented with lower global cognitive performance [12]. The Chronic Renal Insufficiency Cohort (CRIC) study contributed two analyses: Using 6 different cognitive tests, the first substudy with 825 participants reported participants with lower eGFR having lower cognitive scores on most cognitive domains [13]. 
The main CRIC study among 3,591 participants found a higher prevalence of cognitive impairment in those with lower eGFR, independent of traditional vascular risk factors. However, this association was no longer significant after adjustment for hemoglobin concentration [14]. A Taiwanese study of 228 participants yielded lower cognitive function in subjects with CKD [15]. There were two more studies $[16,17]$ which had a cross-sectional and a longitudinal part and those studies are discussed in the following part.

\section{Longitudinal Studies}

Ten prospective studies assessing the relation between CKD and cognitive decline were detected (table 2). The Cardiovascular Health Cognition Study demonstrated that moderate renal insufficiency was associated with a $37 \%$ increased risk of dementia [18]. The Health, Aging, and Body Composition Study demonstrated an increased risk for cognitive impairment when CKD was present. This risk increased with more impaired renal function [19]. The Intervention Project on Cerebrovascular Diseases and Dementia in the Community of Ebersberg (INVADE) study found that moderate-to-severe impaired renal function was associated with incident cognitive impairment after 2 years in a large cohort of elderly subjects [16]. In the Northern Manhattan Study even participants with mildly reduced renal function showed greater cognitive decline compared with participants with normal renal function [20]. The Rush Memory and Aging Project yielded an association of CKD with a more rapid global cognitive decline, i.e. for each eGFR reduction of $15 \mathrm{ml} / \mathrm{min} / 1.73 \mathrm{~m}^{2}$ the increased rate of global cognitive decline had the similar effect of 3 years of aging [21]. The Osaki-Tajiri Project from Japan showed CKD to be significantly associated with incident dementia even after multiple adjustment for cardiovascular risk factors including anemia [22]. The Osteoporotic Fractures in Men Study has been the first study which could not confirm an independent association between CKD and risk of cognitive decline after more than 5 years [17]. Another study in 1,345 participants found an association between reduced cognitive function at follow-up only for baseline albuminuria and only for men, but neither for women nor for eGFR [23]. Among a population-based cohort of 7,839 elderly subjects, no increased risk of cognitive decline was observed after 7 years of follow-up although faster decline of renal function was associated with global cognitive decline and incident dementia with vascular component [24]. The last prospective cohort study consisted of 19,399 adults from the REGARDS study which reported that an impaired eGFR was not associated independently with cognitive impairment if compared with preserved eGFR unless albuminuria was added to the stratification [25].

\section{Meta-Analysis}

For cross-sectional analysis, six studies could be included in the meta-analysis. Three studies could not be included as they did not report OR or HR values or sufficient data to calculate these parameters. For all studies, only the baseline data were used. Meta-analytic pooling using a random-effects model showed that participants with CKD had a significantly increased risk of cognitive impairment compared with those without CKD (OR $1.65,95 \%$ CI 1.32-2.05; p < 0.001) (fig. 2). There was significant heterogeneity amongst the studies (d.f. $=9 ; \mathrm{p}=$ 0.0005).

Regarding longitudinal studies, six studies could be included in the meta-analysis. Four studies could not be included as they did not report OR or HR values or sufficient data to calculate these parameters. Meta-analytic pooling using a random-effects model also revealed that participants with CKD had a significantly increased risk of incident cognitive impairment at follow-up compared with those without CKD (OR 1.39, 95\% CI 1.15-1.68; p < 0.001) (fig. 3). Significant heterogeneity amongst the studies was observed (d.f. $=11 ; \mathrm{p}<0.0001$ ).

Sensitivity Analyses. To investigate the possible differences across studies, we performed sensitivity analyses by grouping studies according to various characteristics such as mean sample size of the study populations (less than/at least 3,000), mean duration of follow-up (less than/at least 3 years) and method used to evaluate cognitive function (MMSE/other). We observed no significant change in the results. In contrast, age and sex contributed significantly to the observed heterogeneity between the studies. Moderate-to-severe CKD (GFR $<45 \mathrm{ml} / \mathrm{min}$ / $1.73 \mathrm{~m}^{2}$ ) is related to an increased risk for cognitive impairment in the cross-sectional (fig. 2) and longitudinal analyses (fig. 3). However, the difference was only significant for the longitudinal studies (OR 1.47 vs. 1.17; fig. 3).

Publication Bias. Funnel plots of effect size versus standard error to investigate possible publication bias were broadly symmetrical, suggesting the absence of publication bias (online suppl. fig. 1 and 2, see www. karger.com/doi/10.1159/000338135). 
Table 1. Cross-sectional studies on the association of CKD and cognitive impairment

\begin{tabular}{|c|c|c|c|c|c|c|}
\hline Study & $\begin{array}{l}\text { Number of } \\
\text { participants }\end{array}$ & $\begin{array}{l}\text { Mean } \\
\text { age } \\
\text { years }\end{array}$ & Cognitive test & $\begin{array}{l}\text { Assessment/ } \\
\text { classification of renal } \\
\text { function }\end{array}$ & $\begin{array}{l}\text { Adjustment for } \\
\text { confounders }\end{array}$ & $\begin{array}{l}\text { Results (risk for cognitive } \\
\text { decline depending on } \\
\text { renal function) }\end{array}$ \\
\hline $\begin{array}{l}\text { Kurella et al., } \\
2005[9]: \\
\text { Heart Estrogen/ } \\
\text { Progestin } \\
\text { Replacement } \\
\text { Study }\end{array}$ & $\begin{array}{l}1,015 \\
\text { (women } \\
\text { with } \\
\text { coronary } \\
\text { artery } \\
\text { disease) }\end{array}$ & 67 & $\begin{array}{l}6 \text { tests (3MS, Trails B, } \\
\text { Boston Naming, Verbal } \\
\text { Fluency, Word List } \\
\text { Memory and Recall) } \\
\text { after } 4 \text { years }\end{array}$ & $\begin{array}{l}\text { MDRD/ } \\
\text { Mild CKD = eGFR } \\
45-60, \text { moderate CKD } \\
=\text { eGFR } 30-44, \text { severe } \\
\text { CKD }=\text { eGFR }<30\end{array}$ & $\begin{array}{l}\text { Age, race, diabetes, } \\
\text { hypertension, CHD, } \\
\text { stroke, education, } \\
\text { exercise, smoking, alcohol, } \\
\text { socioeconomic status }\end{array}$ & $\begin{array}{l}\text { Results for 3MS: } \\
\text { Mild CKD: OR } 1.91 \\
(0.86-4.23) \\
\text { Moderate CKD: OR } 1.80 \\
(0.65-4.97) \\
\text { Severe CKD: OR } 5.01 \\
(1.27-19.7)\end{array}$ \\
\hline $\begin{array}{l}\text { Hailpern et al., } \\
2007 \text { [10]: Third } \\
\text { National Health } \\
\text { and Nutrition } \\
\text { Examination } \\
\text { Survey }\end{array}$ & 4,849 & 36 & $\begin{array}{l}\text { Computerized test } \\
\text { (Simple Reaction } \\
\text { Time, Symbol Digit } \\
\text { Substitution or Serial } \\
\text { Digit Learning) }\end{array}$ & $\begin{array}{l}\text { MDRD/ } \\
\text { Moderate CKD = } \\
\text { eGFR 30-59 }\end{array}$ & $\begin{array}{l}\text { Age, sex, race, education, } \\
\text { medication, smoking, } \\
\text { alcohol, physical activity, } \\
\text { CHD, socioeconomic } \\
\text { status, BMI, hypertension, } \\
\text { diabetes, hyperlipidemia }\end{array}$ & $\begin{array}{l}\text { OR } 2.41 \text { (1.30-5.63) for } \\
\text { concentration; } \\
\text { OR } 2.74 \text { (1.01-7.40 for } \\
\text { visual attention }\end{array}$ \\
\hline $\begin{array}{l}\text { Kurella Tamura } \\
\text { et al., } 2008 \text { [11]: } \\
\text { Reasons for } \\
\text { Geographic } \\
\text { and Racial } \\
\text { Differences } \\
\text { in Stroke } \\
\text { (REGARDS) } \\
\text { Study }\end{array}$ & 23,405 & 65 & 6-Item Screener & $\begin{array}{l}\mathrm{MDRD} / \\
\mathrm{CKD}=\mathrm{eGFR}<60\end{array}$ & $\begin{array}{l}\text { Age, sex, race, education, } \\
\text { region, CHD, stroke, } \\
\text { depression, hypertension, } \\
\text { diabetes, hyperlipidemia, } \\
\text { BMI, smoking }\end{array}$ & OR $1.23(1.06-1.43)$ \\
\hline $\begin{array}{l}\text { Elias et al., } \\
2009 \text { [12]: } \\
\text { Maine-Syracuse } \\
\text { Longitudinal } \\
\text { Study }\end{array}$ & 923 & 63 & $\begin{array}{l}\text { Battery of } 17 \text { tests with } \\
4 \text { cognitive domains }\end{array}$ & $\begin{array}{l}\text { MDRD/ } \\
\mathrm{CKD}=\mathrm{eGFR}<60\end{array}$ & $\begin{array}{l}\text { Age, sex, education, race, } \\
\text { hypertension, diabetes, } \\
\text { BMI, hyperlipidemia, } \\
\text { smoking, stroke }\end{array}$ & $\begin{array}{l}\text { OR } 1.97 \text { (1.25-3.10) for } \\
\text { global composite }\end{array}$ \\
\hline $\begin{array}{l}\text { Yaffe et al., } 2010 \\
\text { [13]: Chronic } \\
\text { Renal } \\
\text { Insufficiency } \\
\text { Cohort } \\
\text { Cognitive Study }\end{array}$ & 825 & 65 & $\begin{array}{l}\text { 3MS, Trails A + B, } \\
\text { Category Fluency, } \\
\text { Buschke Selective } \\
\text { Reminding Test, } \\
\text { Boston Naming }\end{array}$ & $\begin{array}{l}\text { MDRD/ } \\
\text { mild-to-moderate } \\
\text { CKD = eGFR 45-59, } \\
\text { advanced CKD = } \\
\text { eGFR }<30\end{array}$ & $\begin{array}{l}\text { Age, race, sex, education, } \\
\text { BMI, diabetes, } \\
\text { hypertension, depression }\end{array}$ & $\begin{array}{l}\text { OR } 2.0(1.1-3.9) \text { for global } \\
\text { cognition compared with } \\
\text { eGFR 45-59 }\end{array}$ \\
\hline $\begin{array}{l}\text { Yang et al., } 2011 \\
{[15]}\end{array}$ & $\begin{array}{l}228 \\
\text { (only male) }\end{array}$ & 79 & $\begin{array}{l}\text { Cognitive Abilities } \\
\text { Screening Instrument } \\
(\text { CASI) }\end{array}$ & $\begin{array}{l}\text { not described/ } \\
\mathrm{CKD}=\mathrm{eGFR}<60\end{array}$ & Not described & $\begin{array}{l}\text { Positive correlation of } \\
\text { CASI and eGFR }\end{array}$ \\
\hline $\begin{array}{l}\text { Kurella Tamura } \\
\text { et al., } 2011[14]: \\
\text { Chronic Renal } \\
\text { Insufficiency } \\
\text { Cohort Study }\end{array}$ & 3,591 & 58 & $3 \mathrm{MS}$ & $\begin{array}{l}\text { MDRD/ } \\
\text { eGFR <30 with eGFR } \\
45-59 \text { as reference } \\
\text { group }\end{array}$ & $\begin{array}{l}\text { Age, sex, race, ethnicity, } \\
\text { education, clinical site, } \\
\text { stroke, diabetes, } \\
\text { hypertension, smoking, } \\
\text { cholesterol, CHD, PAD }\end{array}$ & $\begin{array}{l}\text { OR } 1.47 \text { (1.05-2.05) but } \\
\text { not significant with } \\
\text { hemoglobin as additional } \\
\text { confounder }\end{array}$ \\
\hline
\end{tabular}

eGFR values are given as $\mathrm{ml} / \mathrm{min} / 1.73 \mathrm{~m}^{2}$.

3MS = Modified MMSE; MDRD = Modification of Diet in Renal Disease CHD = coronary heart disease; PAD = peripheral artery disease.

\section{Discussion}

Our systematic review revealed that most, but not all, cross-sectional [9-15] and longitudinal studies [16, 18-22] suggest an association between cognitive impairment and CKD. However, this review also showed that many cross- sectional and longitudinal studies contain methodological limitations which might explain the divergent results. Specifically, methods for assessing cognitive function showed a great variability between the different cross-sectional and longitudinal studies, for example, some studies used only the MMSE $[14,19,24]$, whereas other trials ap- 
Table 2. Longitudinal studies about the association of CKD and cognitive impairment

\begin{tabular}{|c|c|c|c|c|c|c|c|c|}
\hline Study & $\begin{array}{l}\text { Partici- } \\
\text { pants }\end{array}$ & $\begin{array}{l}\text { Mean } \\
\text { Age }\end{array}$ & $\begin{array}{l}\text { Follow- } \\
\text { up }\end{array}$ & $\begin{array}{l}\text { Cognitive } \\
\text { test }\end{array}$ & $\begin{array}{l}\text { Assessment/ } \\
\text { classification of renal } \\
\text { function }\end{array}$ & $\begin{array}{l}\text { Adjustment for } \\
\text { confounders }\end{array}$ & Results $^{1}$ & Result $^{2}$ \\
\hline $\begin{array}{l}\text { Kurella et al., } \\
2005 \text { [19]: } \\
\text { Health, Aging, } \\
\text { and Body } \\
\text { Composition } \\
\text { Study }\end{array}$ & 3,034 & $\begin{array}{l}74 \\
\text { years }\end{array}$ & 2,4 years & $3 \mathrm{MS}$ & $\begin{array}{l}\text { MDRD at baseline/ } \\
\text { CKD = eGFR }<60 \text { with } \\
\text { two subgroups (eGFR } \\
45-59 \text { and }<45)\end{array}$ & $\begin{array}{l}\text { Age, sex, race, education, } \\
\text { diabetes, medication, } \\
\text { hypertension, } \\
\text { hyperlipidemia, CRP, } \\
\text { IL-6, hematocrit, CHD, } \\
\text { stroke }\end{array}$ & $\begin{array}{l}\text { OR } 1.32 \\
(1.03-1.69) \text { for } \\
\text { eGFR } 45-59 \text {; } \\
\text { OR } 2.43(1.38-4.29) \\
\text { for eGFR < } 45\end{array}$ & Not assessed \\
\hline $\begin{array}{l}\text { Etgen et al., } \\
2009 \text { [16]: } \\
\text { INVADE study }\end{array}$ & 3,697 & $\begin{array}{l}68 \\
\text { years }\end{array}$ & 2 years & $\begin{array}{l}\text { 6-Item } \\
\text { Cognitive } \\
\text { Impairment } \\
\text { Test }\end{array}$ & $\begin{array}{l}\text { CG at baseline/ } \\
\text { mild CKD = eGFR } 45- \\
59, \text { moderate-to-severe } \\
\text { CKD }=\text { eGFR }<45\end{array}$ & $\begin{array}{l}\text { Age, sex, smoking, CHD, } \\
\text { stroke, hypertension, } \\
\text { diabetes, BMI, } \\
\text { hyperlipidemia, alcohol, } \\
\text { physical activity, } \\
\text { depression }\end{array}$ & $\begin{array}{l}\text { Moderate-to- } \\
\text { severe CKD: OR } \\
2.14(1.18-3.87)\end{array}$ & Not assessed \\
\hline $\begin{array}{l}\text { Buchman et al., } \\
2009 \text { [21]: Rush } \\
\text { and Memory } \\
\text { Aging Project }\end{array}$ & 886 & $\begin{array}{l}81 \\
\text { years }\end{array}$ & 3.4 years & $\begin{array}{l}\text { Battery of } 19 \\
\text { tests with } \\
5 \text { cognitive } \\
\text { systems }\end{array}$ & $\begin{array}{l}\text { MDRD at baseline/ } \\
\mathrm{CKD}=\mathrm{eGFR}<60\end{array}$ & $\begin{array}{l}\text { Age, sex, education, BMI, } \\
\text { hemoglobin, physical } \\
\text { activity, social activity, } \\
\text { hypertension, diabetes, } \\
\text { smoking, CHD, stroke, } \\
\text { PAD, depression }\end{array}$ & $\begin{array}{l}\text { Each GFR } \\
\text { reduction of } 15= \\
\text { increased rate of } \\
\text { global cognitive } \\
\text { decline of being } \\
3 \text { years older }\end{array}$ & Not assessed \\
\hline $\begin{array}{l}\text { Sasaki et al., } \\
2011 \text { [22]: } \\
\text { Osaki-Tajiri } \\
\text { Project }\end{array}$ & 497 & $\begin{array}{l}74 \\
\text { years }\end{array}$ & 5 years & $\begin{array}{l}\text { Clinical } \\
\text { Dementia } \\
\text { Rating }\end{array}$ & $\begin{array}{l}\text { not described/ } \\
\text { CKD }=\text { eGFR }<60 \text { or } \\
\text { albuminuria }\end{array}$ & $\begin{array}{l}\text { Age, sex, education, } \\
\text { hypertension, diabetes, } \\
\text { dyslipidemia, CHD, } \\
\text { anemia }\end{array}$ & $\begin{array}{l}\text { OR for conversion } \\
\text { to dementia } 5.3 \\
(1.7-16.2)\end{array}$ & $\begin{array}{l}\text { Not separately } \\
\text { assessed }\end{array}$ \\
\hline $\begin{array}{l}\text { Kurella } \\
\text { Tamura et al., } \\
2011 \text { [25]: } \\
\text { REGARDS }\end{array}$ & 19,399 & $\begin{array}{l}64 \\
\text { years }\end{array}$ & 3.8 years & $\begin{array}{l}\text { 6-Item } \\
\text { Screener }\end{array}$ & $\begin{array}{l}\text { CKD-EPI/ } \\
\text { CKD }=\text { eGFR }<60\end{array}$ & $\begin{array}{l}\text { Age, sex, race, education, } \\
\text { region, hypertension, } \\
\text { diabetes, stroke, CHD, } \\
\text { alcohol, smoking }\end{array}$ & $\begin{array}{l}\text { Not significant for } \\
\text { eGFR }<60\end{array}$ & $\begin{array}{l}\text { OR } 1.30 \\
(1.02-1.66) \text { for } \\
\text { UACR }<10 \mathrm{mg} / \mathrm{g} \\
\text { in eGFR }<60\end{array}$ \\
\hline $\begin{array}{l}\text { Helmer } \\
\text { et al., } 2012 \\
\text { [24]: The Three } \\
\text { City Study }\end{array}$ & 7,839 & $\begin{array}{l}74 \\
\text { years }\end{array}$ & 7 years & MMSE & $\begin{array}{l}\text { CKD-EPI/ } \\
\text { CKD }=\text { eGFR }<60\end{array}$ & $\begin{array}{l}\text { Age, sex, education, } \\
\text { APOE genotype, } \\
\text { hypertension, CHD, } \\
\text { dyslipidemia, diabetes, } \\
\text { smoking, BMI, stroke }\end{array}$ & $\begin{array}{l}\text { Not significant } \\
\text { except for eGFR } \\
\text { decline over first } \\
4 \text { years and } \\
\text { vascular dementia }\end{array}$ & $\begin{array}{l}\text { Borderline risk } \\
\text { for proteinuria }\end{array}$ \\
\hline
\end{tabular}

${ }^{1}$ Risk for cognitive decline depending on renal function. ${ }^{2}$ Risk for cognitive decline based on protein-/albuminuria. All eGFR values are given as $\mathrm{ml} / \mathrm{min} / 1.73 \mathrm{~m}^{2}$. $3 \mathrm{MS}=$ Modified MMSE; TICS = Telephone Interview for Cognitive Status; MDRD = Modification of Diet in Renal Disease; CG = Cockcroft-Gault equation; $\mathrm{CKD}-\mathrm{EPI}=\mathrm{CKD}$ Epidemiology Collaboration equation; $\mathrm{ADL}=$ activities of daily living; $\mathrm{SCr}=$ serum $\mathrm{creatinine}$; $\mathrm{CHD}=\mathrm{coronary}$ heart disease; $\mathrm{PAD}=$ peripheral artery disease; $\mathrm{UACR}=$ urine albumin-creatinine ratio. 


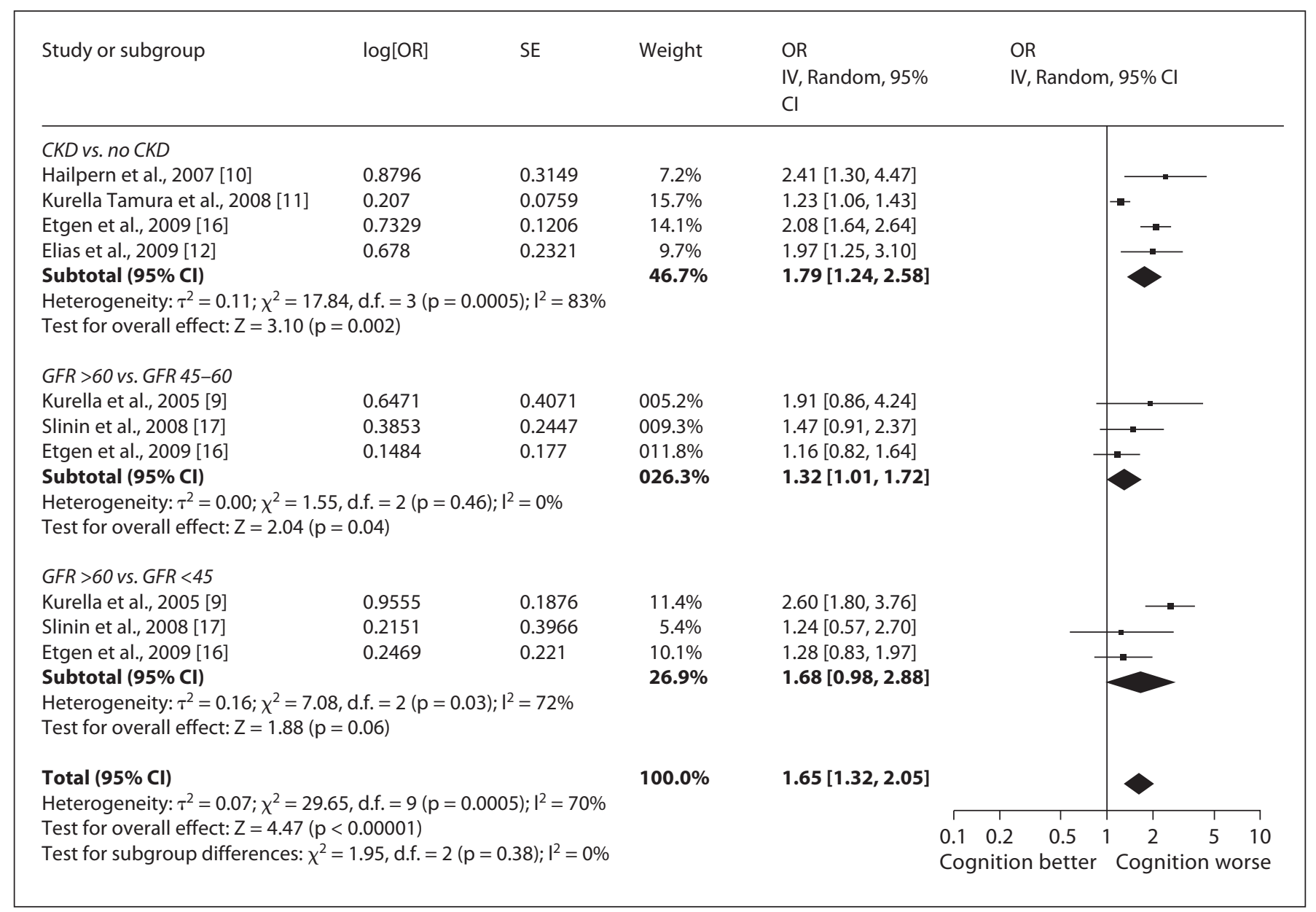

Fig. 2. Forest plot of cross-sectional studies assessing the association of CKD and cognitive decline.

plied a battery of multi-domain cognitive tests for episodic memory, semantic memory, working memory, perceptual speed and visuospatial abilities $[9,12,21]$. The study population varied from gender-specific (e.g. only women with coronary heart disease [9] or only men [17]) to community-dwelling participants [16]. The mean age of the study population ranged from 36 [10] to 81 years [21]. Several methods of defining CKD (i.e. slope of creatinine or different prediction equations: Modification of Diet in Renal Disease (MDRD), Cockcroft-Gault, CKD Epidemiology Collaboration equation) have been used, as it remains unclear which is the best method for assessing CKD in elderly cognitively impaired patients [26]. The inclusion of different potential confounders is another critical point. A number of studies have used only a few confounders, whereas others considered many confounding factors (tables 1,2). Some confounders that received in- creasing attention over recent years (e.g. depression and physical activity) have not been included.

Our meta-analysis is the first that assessed the relation between CKD and cognitive decline and included 54,779 participants of cross-sectional and longitudinal studies. This meta-analysis revealed that participants with CKD had a significantly increased risk of cognitive impairment compared with those without CKD either in crosssectional or in longitudinal studies. This association remained present independent of the stage of CKD and was even stronger in the group with moderate-to-severe CKD (GFR $<45 \mathrm{ml} / \mathrm{min} / 1.73 \mathrm{~m}^{2}$ ) compared with mild-to-moderate CKD (GFR 45-60 ml/min/1.73 $\mathrm{m}^{2}$ ). Further sensitivity analyses by grouping studies according to various characteristics such as mean sample size of the study populations (less than/at least 3,000), mean duration of follow-up (less than/at least 3 years) and method used to 


\begin{tabular}{|c|c|c|c|c|c|c|}
\hline Study or subgroup & $\log [\mathrm{OR}]$ & SE & Weight & $\begin{array}{l}\text { OR } \\
\text { IV, Random, 95\% CI }\end{array}$ & \multicolumn{2}{|c|}{$\begin{array}{l}\text { OR } \\
\text { IV, Random, } 95 \% \mathrm{Cl}\end{array}$} \\
\hline \multicolumn{7}{|l|}{$C K D$ vs. no $C K D$} \\
\hline Etgen et al., 2009 [16] & 0.7938 & 0.1638 & $9.7 \%$ & $2.21[1.60,3.05]$ & & 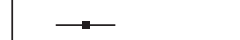 \\
\hline Sasaki et al., 2011 [22] & 1.6677 & 0.5802 & $2.3 \%$ & $5.30[1.70,16.52]$ & & $\longrightarrow$ \\
\hline \multicolumn{7}{|c|}{$\begin{array}{l}\text { Heterogeneity: } \tau^{2}=0.20 ; \chi^{2}=2.10, \text { d.f. }=1(p=0.15) ; 1^{2}=52 \% \\
\text { Test for overall effect: } Z=2.64(p=0.008)\end{array}$} \\
\hline \multicolumn{7}{|l|}{ GFR $>60$ vs. GFR 45-60 } \\
\hline Kurella et al., 2005 [19] & 0.27 & 0.1178 & $11.2 \%$ & $1.31[1.04,1.65]$ & & $\leftarrow$ \\
\hline Slinin et al., 2008 [17] & 0.0583 & 0.1187 & $11.2 \%$ & $1.06[0.84,1.34]$ & & \\
\hline Etgen et al., 2009 [16] & 0.1133 & 0.2471 & $7.2 \%$ & $1.12[0.69,1.82]$ & &. \\
\hline Kurella Tamura et al., 2011 [25] & 0.0862 & 0.1092 & $11.5 \%$ & $1.09[0.88,1.35]$ & & $t=-$ \\
\hline Helmer et al., 2011 [24] & 0.2467 & 0.1338 & $10.7 \%$ & $1.28[0.98,1.66]$ & & 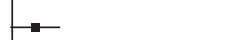 \\
\hline \multicolumn{7}{|c|}{$\begin{array}{l}\text { Heterogeneity: } \tau^{2}=0.00 ; \chi^{2}=2.51, \text { d.f. }=4(p=0.64) ; I^{2}=0 \% \\
\text { Test for overall effect: } Z=2.69(p=0.007)\end{array}$} \\
\hline \multicolumn{7}{|l|}{ GFR $>60$ vs. GFR $<45$} \\
\hline Kurella et al., 2005 [19] & 1.0508 & 0.2565 & $6.9 \%$ & $2.86[1.73,4.73]$ & & \\
\hline Slinin et al., 2008 [17] & 0.1655 & 0.2312 & $7.6 \%$ & $1.18[0.75,1.86]$ & & \\
\hline Etgen et al., 2009 [16] & 0.7608 & 0.3037 & $5.8 \%$ & $2.14[1.18,3.88]$ & & \\
\hline Kurella Tamura et al., 2011 [25] & 0.0488 & 0.1452 & $10.3 \%$ & $1.05[0.79,1.40]$ & & - \\
\hline Helmer et al., 2011 [24] & -0.0217 & 0.316 & $5.5 \%$ & $0.98[0.53,1.82]$ & & \\
\hline \multicolumn{7}{|c|}{$\begin{array}{l}\text { Heterogeneity: } \tau^{2}=0.16 ; \chi^{2}=15.22, \text { d.f. }=4(p=0.004) ; I^{2}=74 \% \\
\text { Test for overall effect: } Z=1.84(p=0.07)\end{array}$} \\
\hline Total $(95 \% \mathrm{Cl})$ & & & $100.0 \%$ & $1.39[1.15,1.68]$ & & \\
\hline \multicolumn{7}{|c|}{$\begin{array}{l}\text { Heterogeneity: } \tau^{2}=0.07 ; \chi^{2}=37.55, \text { d.f. }=11(p<0.0001) ; I^{2}=71 \% \\
\text { Test for overall effect: } Z=3.42(p=0.00006) \\
\text { Test for subgroup differences: } \chi^{2}=5.90, \text { d.f. }=2(p=0.5) ; I^{2}=66.1 \%\end{array}$} \\
\hline
\end{tabular}

Fig. 3. Forest plot of longitudinal studies assessing the association of $\mathrm{CKD}$ and cognitive decline.

evaluate cognitive function (MMSE/other) yielded no significant differences across studies.

Several factors may help to explain the association between CKD and cognitive decline. Patients with CKD have a higher prevalence of subclinical cerebrovascular disease than the general population [27]. Beyond traditional vascular risk factors, which include hypertension, diabetes mellitus, hypercholesterolemia, cigarette smoking and cardiovascular disease, there are other non-traditional vascular risk factors that may be associated with cognitive impairment. These factors comprise hyperhomocysteinemia, hemostatic abnormalities or hypercoaguable states (e.g. Factor V Leiden mutation, abnormalities in the thrombin/antithrombin complex or of the tissue type plasminogen activator), inflammation, and oxidative stress $[6,28]$. In addition, nonvascular risk factors play a role in the development of cognitive decline: Anemia in CKD has been associated with cognitive impairment [29] and neuropsychological and neurophysiological tests have shown improvement with the treatment of anemia in CKD [30]. Multiple medications in CKD patients and uncertainty of the optimal dosing make these patients more susceptible to side effects and interactions between medications. Sleep disturbances are frequent in patients with CKD resulting in an impaired concentration, excessive daytime fatigue and possibly cognitive dysfunction [31].

Based on the pathophysiological mechanisms of the association between CKD and cognitive decline, some studies have tested intervention strategies to improve cognitive function. In a randomized placebo-controlled trial of 659 advanced CKD patients, treatment with high 
daily doses of B vitamins was effective in lowering initially high homocysteine levels, but did not affect cognitive outcomes after one year [32]. Large randomized trials of anemia improvement using erythropoietin in CKD patients did not specifically assess cognitive function, but one study showed an association with an increased risk of stroke which is a risk factor for dementia [33]. The ongoing largest blood pressure study launched by the $\mathrm{NIH}$, the Systolic Blood Pressure Intervention Trial (SPRINT), will assess with a multi-center randomized design whether maintaining blood pressure levels lower than current recommendations further reduces the risk of age-related cognitive decline in patients with and without CKD [34].

There are some limitations of this review. Albuminuria or proteinuria has recently been proposed as a complementary risk factor of cognitive decline, however, the available data did not allow us perform a separate metaanalysis to assess the relationship between kidney damage with preserved kidney function (i.e. albuminuria) and cognitive function. Due to the lack of eGFR being reported as a continuous variable and the great variability among cognitive tests we were not able to examine a relationship change of MMSE by unit decrements of eGFR. In summary, our results confirm the increasing evidence of CKD as an independent risk factor for cognitive decline. This may also highlight the importance of preventive strategies including the identification of risk factors like CKD among patients with cognitive decline.

\section{Disclosure Statement}

There are no conflicts of interest to declare.

\section{References}

1 Fratiglioni L, Qiu C: Prevention of cognitive decline in ageing: dementia as the target, delayed onset as the goal. Lancet Neurol 2011; 10:778-779.

-2 Gauthier S, Reisberg B, Zaudig M, Petersen RC, Ritchie K, Broich K, Belleville S, Brodaty $\mathrm{H}$, Bennett D, Chertkow H, Cummings JL, de Leon M, Feldman H, Ganguli M, Hampel $\mathrm{H}$, Scheltens P, Tierney MC, Whitehouse P, Winblad B: Mild cognitive impairment. Lancet 2006;367:1262-1270.

-3 Petersen RC: Clinical practice. Mild cognitive impairment. N Engl J Med 2011;364: 2227-2234.

4 Etgen T, Sander D, Bickel H, Förstl H: Mild cognitive impairment and dementia: the importance of modifiable risk factors. Dtsch Arztebl Int 2011;108:743-750.

5 Barnes DE, Yaffe K: The projected effect of risk factor reduction on Alzheimer's disease prevalence. Lancet Neurol 2011;10:819828.

6 Madero M, Gul A, Sarnak MJ: Cognitive function in chronic kidney disease. Semin Dial 2008;21:29-37.

7 Etgen T, Bickel H, Förstl H: Metabolic and endocrine factors in mild cognitive impairment. Ageing Res Rev 2010;9:280-288.

8 Stroup DF, Berlin JA, Morton SC, Olkin I, Williamson GD, Rennie D, Moher D, Becker BJ, Sipe TA, Thacker SB: Meta-analysis of observational studies in epidemiology: a proposal for reporting. Meta-analysis Of Observational Studies in Epidemiology (MOOSE) group. Jama 2000;283:2008-2012.

Chronic Kidney Disease and Cognitive Impairment
-9 Kurella M, Yaffe K, Shlipak MG, Wenger NK, Chertow GM: Chronic kidney disease and cognitive impairment in menopausal women. Am J Kidney Dis 2005;45:66-76.

10 Hailpern SM, Melamed ML, Cohen HW, Hostetter TH: Moderate chronic kidney disease and cognitive function in adults 20 to 59 years of age: Third National Health and Nutrition Examination Survey (NHANES III). J Am Soc Nephrol 2007;18:2205-2213.

11 Kurella Tamura M, Wadley V, Yaffe K, McClure LA, Howard G, Go R, Allman RM, Warnock DG, McClellan W: Kidney function and cognitive impairment in US adults: the Reasons for Geographic and Racial Differences in Stroke (REGARDS) Study. Am J Kidney Dis 2008;52:227-234.

12 Elias MF, Elias PK, Seliger SL, Narsipur SS, Dore GA, Robbins MA: Chronic kidney disease, creatinine and cognitive functioning. Nephrol Dial Transplant 2009;24:24462452.

13 Yaffe K, Ackerson L, Kurella Tamura M, Le Blanc P, Kusek JW, Sehgal AR, Cohen D, Anderson C, Appel L, Desalvo K, Ojo A, Seliger S, Robinson N, Makos G, Go AS: Chronic kidney disease and cognitive function in older adults: findings from the chronic renal insufficiency cohort cognitive study. J Am Geriatr Soc 2010;58:338-345.

14 Kurella Tamura M, Xie D, Yaffe K, Cohen DL, Teal V, Kasner SE, Messe SR, Sehgal AR, Kusek J, DeSalvo KB, Cornish-Zirker D, Cohan J, Seliger SL, Chertow GM, Go AS: Vascular risk factors and cognitive impairment in chronic kidney disease: the Chronic Renal Insufficiency Cohort (CRIC) study. Clin J Am Soc Nephrol 2011;6:248-256.
15 Yang AC, Tsai SJ, Yeh HL, Chen JY, Liou YJ Hwang JP, Hong CJ: Association between renal function and cognitive performance in elderly community-dwelling men without dementia. J Am Geriatr Soc 2011;58:2046-2048.

16 Etgen T, Sander D, Chonchol M, Briesenick C, Poppert H, Förstl H, Bickel H: Chronic kidney disease is associated with incident cognitive impairment in the elderly: the INVADE study. Nephrol Dial Transplant 2009; 24:3144-3150.

17 Slinin Y, Paudel ML, Ishani A, Taylor BC, Yaffe K, Murray AM, Fink HA, Orwoll ES, Cummings SR, Barrett-Connor E, Jassal S, Ensrud KE: Kidney function and cognitive performance and decline in older men. J Am Geriatr Soc 2008;56:2082-2088.

18 Seliger SL, Siscovick DS, Stehman-Breen CO, Gillen DL, Fitzpatrick A, Bleyer A, Kuller LH: Moderate renal impairment and risk of dementia among older adults: the Cardiovascular Health Cognition Study. J Am Soc Nephrol 2004;15:1904-1911.

19 Kurella M, Chertow GM, Fried LF, Cummings SR, Harris T, Simonsick E, Satterfield $\mathrm{S}$, Ayonayon H, Yaffe K: Chronic kidney disease and cognitive impairment in the elderly: the health, aging, and body composition study. J Am Soc Nephrol 2005; 16:2127-2133.

20 Khatri M, Nickolas T, Moon YP, Paik MC, Rundek T, Elkind MS, Sacco RL, Wright CB: CKD associates with cognitive decline. J Am Soc Nephrol 2009;20:2427-2432.

21 Buchman AS, Tanne D, Boyle PA, Shah RC, Leurgans SE, Bennett DA: Kidney function is associated with the rate of cognitive decline in the elderly. Neurology 2009;73:920927. 
22 Sasaki Y, Marioni R, Kasai M, Ishii H, Yamaguchi S, Meguro K: Chronic kidney disease: a risk factor for dementia onset: a population-based study. The Osaki-Tajiri Project. J Am Geriatr Soc 2011;59:1175-1181.

23 Jassal SK, Kritz-Silverstein D, Barrett-Connor E: A prospective study of albuminuria and cognitive function in older adults: the Rancho Bernardo study. Am J Epidemiol 2010;171:277-286.

-24 Helmer C, Stengel B, Metzger M, Froissart M, Massy ZA, Tzourio C, Berr C, Dartigues JF: Chronic kidney disease, cognitive decline, and incident dementia: the 3C Study. Neurology 2011;77:2043-2051.

25 Kurella Tamura M, Muntner P, Wadley V, Cushman M, Zakai NA, Bradbury BD, Kissela B, Unverzagt F, Howard G, Warnock D, McClellan W: Albuminuria, kidney function, and the incidence of cognitive impairment among adults in the United States. Am J Kidney Dis 2011;58:756-763.
26 Alagiakrishnan K, Senthilselvan A: Low agreement between the modified diet and renal disease formula and the Cockcroft-Gault formula for assessing chronic kidney disease in cognitively impaired elderly outpatients. Postgrad Med 2010;122:41-45.

27 Seliger SL, Sarnak MJ: Subclinical vascular disease of the brain in dialysis patients. Am J Kidney Dis 2007;50:8-10.

28 Breteler MM: Vascular risk factors for Alzheimer's disease: an epidemiologic perspective. Neurobiol Aging 2000;21:153-160.

29 Eisenstaedt R, Penninx BW, Woodman RC: Anemia in the elderly: current understanding and emerging concepts. Blood Rev 2006; 20:213-226.

30 Stivelman JC: Benefits of anaemia treatment on cognitive function. Nephrol Dial Transplant 2000;15(suppl 3):29-35.
1 Iliescu EA, Coo H, McMurray MH, Meers CL, Quinn MM, Singer MA, Hopman WM: Quality of sleep and health-related quality of life in haemodialysis patients. Nephrol Dial Transplant 2003;18:126-132.

32 Brady CB, Gaziano JM, Cxypoliski RA, Guarino PD, Kaufman JS, Warren SR, Hartigan P, Goldfarb DS, Jamison RL: Homocysteine lowering and cognition in CKD: the Veterans Affairs homocysteine study. Am J Kidney Dis 2009;54:440-449.

33 Pfeffer MA, Burdmann EA, Chen CY, Cooper ME, de Zeeuw D, Eckardt KU, Feyzi JM, Ivanovich P, Kewalramani R, Levey AS, Lewis EF, McGill JB, McMurray JJ, Parfrey P, Parving HH, Remuzzi G, Singh AK, Solomon SD, Toto R: A trial of darbepoetin alfa in type 2 diabetes and chronic kidney disease. N Engl J Med 2009;361:2019-2032.

34 National Institutes of Health: NIH Launches Multicenter Clinical Trial to Test Blood Pressure Strategy 2w009. http:/www.nih.gov/ news/health/oct2009/nhlbi-29.htm. 